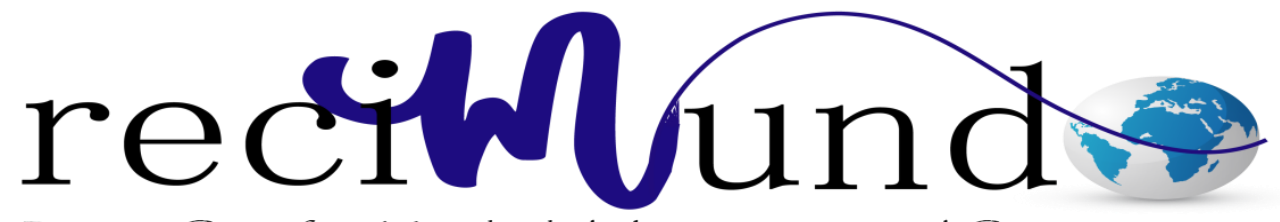

Revista Científica Mundo de la Investigación y el Conocimiento

Liseth Pierina Pinto López ${ }^{\text {a }}$; Mercy Nathaly Alarcón Sánchez ${ }^{\text {; }}$ S Segundo Pacherres Seminario ${ }^{c}$

Determinar plan nutricional en diabetes mellitus tipo 2 infantil y diseñar una guía didáctica alimentaria

Revista Científica Mundo de la Investigación y el Conocimiento. Vol. 2 núm., especial, mayo, ISSN: 2588-073X, 2018, pp. 464-477

DOI: 10.26820/recimundo/2.esp.2018.464-477

Editorial Saberes del Conocimiento

Recibido: 05/12/2017

Aceptado: 15/02/2018

a. Universidad de Guayaquil.

b. Universidad de Guayaquil.

c. Universidad de Guayaquil. 


\section{Determinar plan nutricional en diabetes mellitus tipo 2 infantil y diseñar una \\ guía didáctica alimentaria}

Vol. 2, núm. Esp., (2018)

Liseth Pierina Pinto López; Mercy Nathaly Alarcón Sánchez; Segundo Pacherres Seminario

\section{RESUMEN}

La diabetes es una de las enfermedades crónicas más frecuentes a nivel mundial, en el pasado la diabetes mellitus tipo 2 era diagnosticada mayormente en adultos, actualmente esta enfermedad afecta más en niños y adolescentes, debido al incremento de sobrepeso y obesidad que hay en el país, esto ha representado un problema en la salud pública y en la infancia, con consecuencias potencialmente graves. En el Departamento de Diabetes Care del Laboratorio Roche se atienden aproximadamente a 65 personas por mes en la que el $40 \%$ de la población son niños; a pesar de que la enfermedad no tiene cura puede ser tratada mediante cuatro pilares fundamentales (alimentación, actividad física, autocontrol, medicamentos) que forman parte dentro del tratamiento en la diabetes, lo que este trabajo de investigación tiene como objetivo, es determinar plan nutricional en diabetes mellitus tipo 2 infantil y diseñar guía didáctica alimentaria año 2013 - 2014, teniendo como propuesta la creación de una guía didáctica alimentaria. La muestra estuvo conformada por 138 pacientes, la cual se aplicó un instrumento de recolección de datos (recordatorio de 24 horas, anamnesis alimentaria, etc.), la cual permitió recoger datos acerca de la forma como se alimentan, antecedentes personales, familiares. Así como datos antropométricos (peso, talla, IMC) siendo de tipo longitudinal se trabajó en el mes de septiembre a enero. El estudio revelo que el $51 \%$ de los pacientes con diabetes mellitus tipo 2 infantil se encuentran en los niños lo cual se realizó una guía didáctica alimentaria, en la cual consta de recomendaciones, semáforo de alimentos, las porciones correctas que se debe consumir, conjunto con actividad física como propuesta de la investigación, llegando a resultados esperados en la aceptación de charlas, talleres y sobretodo en el control de glicemia de parte de los padres.

Descriptores: Estado Nutricional, Diabetes Tipo 2, Plan Alimentario. 


\title{
Determinar plan nutricional en diabetes mellitus tipo 2 infantil y diseñar una guía didáctica alimentaria
}

Vol. 2, núm. Esp., (2018)

Liseth Pierina Pinto López; Mercy Nathaly Alarcón Sánchez; Segundo Pacherres Seminario

\begin{abstract}
Diabetes is one of the most frequent chronic diseases worldwide, in the past diabetes mellitus type 2 was diagnosed mostly in adults, currently this disease affects more in children and adolescents, due to the increase in overweight and obesity in the country This has represented a problem in public health and in childhood, with potentially serious consequences. In the Diabetes Care Department of the Roche Laboratory approximately 65 people are attended per month in which $40 \%$ of the population are children; Although the disease has no cure can be treated by four fundamental pillars (food, physical activity, self-control, drugs) that are part of the treatment in diabetes, what this research has as an objective, is to determine nutritional plan in type 2 diabetes mellitus in children and design a didactic food guide for 2013 - 2014, with the proposal of creating a didactic food guide. The sample consisted of 138 patients, which was applied a data collection instrument (24-hour reminder, food history, etc.), which allowed to collect data about the way they eat, personal history, family. As well as anthropometric data (weight, height, BMI) being of longitudinal type was worked in the month of September to January. The study revealed that $51 \%$ of patients with type 2 diabetes mellitus in children are found in children, which was a didactic food guide, which consists of recommendations, food semaphore, the correct portions to be consumed, set with physical activity as a proposal of the research, reaching expected results in the acceptance of talks, workshops and especially in the control of blood glucose by parents.
\end{abstract}

Keywords: Nutritional Status, Type 2 Diabetes, Food Plan. 


\section{Determinar plan nutricional en diabetes mellitus tipo 2 infantil y diseñar una \\ guía didáctica alimentaria}

Vol. 2, núm. Esp., (2018)

Liseth Pierina Pinto López; Mercy Nathaly Alarcón Sánchez; Segundo Pacherres Seminario

\section{Introducción.}

Se debe reconocer, que la diabetes mellitus todavía sigue siendo una enfermedad difícil, crónica e incurable; pero perfectamente tratable y controlable con las respectivas atenciones y cuidados. (1)

Buscar sus causas, conseguir una adecuada nutrición y un buen control metabólico, combatir las complicaciones que se presentan, descubrir un correcto tratamiento para mejorar la calidad de vida de los niños con diabetes, han sido y siguen siendo los objetivos de la historia de la medicina. En los siglos anteriores la diabetes mellitus tipo 2 solo se presentaba en los adultos, en la actualidad hay gran número de niños con diabetes tipo 2, los factores son muchos; hereditario, sobrepeso, obesidad, falta de actividad física y malos hábitos alimentarios. (2)

La diabetes mellitus es una enfermedad metabólica que se caracteriza por presencias de hiperglucemia (aumento de la glucosa en la sangre). Esto es resultado de defectos en el páncreas, debido a que al no producir suficiente insulina o nada de insulina. Las células o son resistentes a los efectos de la insulina (o ésta no es suficiente), entonces la glucosa se acumula en la sangre, a niveles que no son saludables y pueden ser peligrosos para la salud. Con el tiempo, la diabetes pone en riesgo al cuerpo del niño, adolescente o adulto, de sufrir complicaciones asociadas con la diabetes como: enfermedades cardíacas, ceguera, neuropatías, problemas renales, entre otros. La diabetes mellitus es producida por muchas causas: hereditarias, virales y nutricionales, estas ocasionan una reducción, deficiencia, carencia o destrucción autoinmune de las células beta o resistencia periférica a la acción de la insulina en sus tejidos, lo que dará lugar a anormalidades en el metabolismo de hidratos de carbono, grasas y proteínas. (3) 


\section{Determinar plan nutricional en diabetes mellitus tipo 2 infantil y diseñar una guía didáctica alimentaria}

Vol. 2, núm. Esp., (2018)

Liseth Pierina Pinto López; Mercy Nathaly Alarcón Sánchez; Segundo Pacherres Seminario

La prevalencia de Diabetes Mellitus Tipo 2 infantil cada año ha venido en aumento, debido al incremento de obesidad, la alta frecuencia de sedentarismo que presenta la población, el antecedente familiar también es un factor en este tipo de diabetes mellitus. (4)

Según la Agencia Pública de Noticias del Ecuador y Suramérica (Ándes) el 44\% de los casos mundiales de diabetes mellitus infantil son atribuibles al sobrepeso y obesidad. Ha existido el concepto de que un "niño gordito es un niño saludable", este pensamiento ha estado muy arraigado en el personal de salud y en los centros de cuidado de niños. A medida que se observan mejor los riesgos de la obesidad para la salud, se impone un cambio en estos pensamientos a nivel familiar, y sanitario. (5)

Aproximadamente $60 \%$ de los niños y adolescentes con sobrepeso tienen al menos un factor de riesgo adicional para enfermedades cardiovasculares, tales como elevada tensión arterial, hiperlipidemia o hiperinsulinemia. Más del 25\% tiene 2 o más de esos factores de riesgo. La diabetes tipo 2 se estima que aparece entre el 8 al $45 \%$ de todos los nuevos casos de diabetes en niños y adolescentes.

En esta investigación se tiene como propuesta que los niños con diabetes mellitus tipo 2 mejoren la calidad de vida, dando pautas, recomendaciones nutricionales, modificando sus hábitos en la alimentación, a través de la guía didáctica alimentaria que se va a realizar, fomentar la actividad física para disminuir el sobrepeso y la obesidad debido a que estos son uno de los factores principales por el cual se relaciona la diabetes mellitus tipo 2 infantil, se pretende que mediante charlas educativas para padres junto con sus hijos tengan mayor conocimiento acerca de la enfermedad y su tratamiento. 


\section{Determinar plan nutricional en diabetes mellitus tipo 2 infantil y diseñar una}

guía didáctica alimentaria

Vol. 2, núm. Esp., (2018)

Liseth Pierina Pinto López; Mercy Nathaly Alarcón Sánchez; Segundo Pacherres Seminario El propósito no solo es informar si no lograr, un cambio de conducta alimentaria en el entorno familiar.

El objetivo de la presente investigación es determinar plan nutricional en Diabetes Mellitus tipo 2 infantil en el Departamento de Diabetes Care de Laboratorio Roche año 20132014.

\section{Metodología.}

Diseño de la investigación

De acuerdo al problema y a los objetivos planteados; la presente investigación es de tipo cuantitativo, a través de registros antropométricos, anamnesis alimentaria se obtendrá información que se requiere para su total análisis y así poder llegar a nuestro objetivo, que es diseñar una guía didáctica alimentaria con el fin de que los padres de familia de niños con diabetes tengan mayor conocimiento sobre la enfermedad y mejorar el estilo de vida del niño, la prevención de complicaciones y sobre todo llevar una mejor calidad de vida.

Tipo de la investigación

Para la presente investigación se utilizara material bibliográfico y documental que permitirá el soporte de argumentación científica.

Investigación bibliográfica: Es la utilización de información obtenida a través de libros, revistas, sucesos científicos, sobre el tema a tratar en esta investigación 


\section{Determinar plan nutricional en diabetes mellitus tipo 2 infantil y diseñar una guía didáctica alimentaria}

Vol. 2, núm. Esp., (2018)

Liseth Pierina Pinto López; Mercy Nathaly Alarcón Sánchez; Segundo Pacherres Seminario

\section{Nivel de estudio}

Debido a las características de esta investigación el nivel de estudio será de campo ya que se aplicaran técnicas necesarias para el análisis de la muestra.

\section{Muestra}

Parte o fracción representativa de una población, universo o colectivo que ha sido obtenida con el fin de investigar ciertas características del mismo. El problema principal consiste en asegurar que el subconjunto sea representativo de la población, de manera que permita generalizar al universo los resultados obtenidos sobre la muestra.

Al aplicar la formula a la población, la muestra será la siguiente:

\begin{tabular}{|c|c|}
\hline MUESTRA & CANTIDAD \\
\hline Diabetes tipo II & 138 \\
\hline TOTAL & 138 \\
\hline
\end{tabular}

Instrumento de la investigación

El instrumento que se utilizó en el siguiente proyecto fue: "la observación”, para revisar toda la información necesaria, para verificar los planteamientos formulados e iniciar el trabajo.

La observación permite sugerir ideas, para motivar, conducir y formular los datos según la necesidad observada. 


\section{Determinar plan nutricional en diabetes mellitus tipo 2 infantil y diseñar una \\ guía didáctica alimentaria}

Vol. 2, núm. Esp., (2018)

Liseth Pierina Pinto López; Mercy Nathaly Alarcón Sánchez; Segundo Pacherres Seminario

\section{Planes de alimentación}

Permite recopilar datos obtenidos a través de las fichas emitidas a cada uno de los integrantes de nuestra muestra, donde se debe investigar. La ficha se caracteriza por que con muestra estudiada al llenar un cuestionario de preguntas sobre el tema propuesto con anterioridad.

\section{Menús diarios}

Nos brindara el punto de salida de la investigación ya que se usaran los menús ya establecidos para así analizarlos y determinando su aceptabilidad con respecto a la población en los que se los emplea.

\section{Encuesta alimentaria}

Nos dará una idea de cómo se encuentran los hábitos de alimentación en los niños y poder intervenir de manera personalizada abarcando en el buen aporte nutricional.

Anamnesis alimentaria: en la aplicación de la guía alimentaria.

Procedimiento de recolección de la investigación

Para esta investigación se realizó mediciones antropométricas en la cual se valoró el estado nutricional de los niños, así mismo se realizó circunferencia de la cintura para valorar a niños que son propensos a padecer enfermedades cardiovasculares de acuerdo a los valores arrojados. 


\section{Determinar plan nutricional en diabetes mellitus tipo 2 infantil y diseñar una guía didáctica alimentaria}

Vol. 2, núm. Esp., (2018)

Liseth Pierina Pinto López; Mercy Nathaly Alarcón Sánchez; Segundo Pacherres Seminario

Se realizó Charlas Educativas a los padres de los niños con diabetes, en la cual conto con temas de gran importancia para el tratamiento de la diabetes se abarco temas como: Importancia de la Nutrición, Importancia del Autocontrol, Comiendo Sano con la finalidad de que los padres con niños diabéticos sepan que tan importante es llevar los cuatro pilares fundamentales de la diabetes en el tratamiento, estos pilares son: alimentación, autocontrol, ejercicios y medicamentos. Así mismo se procedió a realizar encuestas dietéticas las cuales consta de; Frecuencia de Consumo de alimentos que nos permite conocer como ha sido la alimentación del niño, nos ayudara a ver cuáles son los alimentos que mayormente consume y si estos son beneficiosos para la salud; Recordatorio 24 Horas, esta nos indica conocer cuáles fueron las comidas que realizo en el día; Anamnesis Alimentaria mediante esta podremos conocer hábitos alimentarios, sus horarios de comida, sus costumbres.

\section{Procesamiento y análisis}

El proceso de información se coloca desde el índice, el capítulo I hasta el capítulo V se elaboró mediante el sistema de computación de Microsoft, Excel, mediante el proceso de cuadros y gráficos de los datos y se analizan en el correspondiente capítulo.

Luego recolectados los datos se utilizó el programa Excel. Se realizaron cuadros de frecuencias de toda la variable. 


\section{Determinar plan nutricional en diabetes mellitus tipo 2 infantil y diseñar una}

guía didáctica alimentaria

Vol. 2, núm. Esp., (2018)

Liseth Pierina Pinto López; Mercy Nathaly Alarcón Sánchez; Segundo Pacherres Seminario

\section{Resultados.}

Análisis e interpretación de los resultados

Los resultados que se obtuvieron con la investigación, mediante los indicadores, parámetros antropométricos, encuesta y charlas, la cual se aplicó a 138 niños con diabetes mellitus tipo 2 y a los respectivos padres de familia.

En la siguiente hoja se observara las preguntas con su respectivo cuadro, gráficos y el análisis de cada uno, lo cual explican y detallan los resultados obtenidos que han sido favorables para esta investigación.

\section{Grupo Etario}

El grupo etario que presenta mayor prevalencia de diabetes mellitus tipo 2 corresponde al grupo de edades entre 8 a 9 años de edad con un $43 \%$ a diferencia del grupo de edades de 10 a 11 años con $35 \%$.

\section{Género}

De acuerdo a la encuesta realizada se obtuvo como resultado que había mayor cantidad de niños con diabetes representando un $51 \%$ de la muestra a diferencia de las niñas que tienen un $49 \%$, esto se debe a muchos factores.

$I M C$ 


\section{Determinar plan nutricional en diabetes mellitus tipo 2 infantil y diseñar una guía didáctica alimentaria}

Vol. 2, núm. Esp., (2018)

Liseth Pierina Pinto López; Mercy Nathaly Alarcón Sánchez; Segundo Pacherres Seminario

Los resultados que se obtuvieron al realizar el IMC a los niños arrojaron que la mayoría presentaba sobrepeso teniendo como resultado un $47 \%$ y un $40 \%$ se encontraba en su peso normal, esto se debe a que existen muchos niños con muy malos hábitos alimentarios y no hay un control.

\section{Indicé Glicémico}

Se observa que no hay control en la alimentación del niño puesto que en los exámenes una mayor cantidad de niños presentan un índice glicémico alto de 120 a $130 \mathrm{mg} / \mathrm{dl}$ dando como resultado un $46 \%$ de la muestra y un $35 \%$ de los niños se encuentran dentro del rango normal en los niveles de glicemia.

\section{Hábitos Alimentarios}

Los resultados arrojados en la encuesta realizada a los padres de familia con respecto a los hábitos alimentarios de los niños indican que el $44 \%$ de los niños tienen malos hábitos alimentarios referentes a la ingesta de Carbohidratos y que el 37\% es regular.

\section{Educación Diabetológica}

Mediante las evaluaciones necesarias se determinó que 85 padres de familia mostraron efectos beneficiosos para la salud del niño después de la educación diabetológica recibida con un $61 \%$ y un $39 \%$ fueron adecuados siendo igual una respuesta muy buena.

\section{Guía Didáctica Alimentaria}




\section{Determinar plan nutricional en diabetes mellitus tipo 2 infantil y diseñar una \\ guía didáctica alimentaria}

Vol. 2, núm. Esp., (2018)

Liseth Pierina Pinto López; Mercy Nathaly Alarcón Sánchez; Segundo Pacherres Seminario

De acuerdo a la recolección de datos después de haber brindado la guía didáctica alimentaria a los padres de familia para que el niño siga llevando su estilo de vida correcto se obtuvo como resultado que el $63 \%$ llevaba un mejor estilo de vida, tenían mejores hábitos alimentarios y seguían las recomendaciones dadas.

\section{Actividad Física}

El resultado de las encuestas realizadas indica que el $32 \%$ de los niños no realizan ejercicios tan seguidos y que el $52 \%$ no realiza ejercicios puesto que solo pasa en casa viendo tv, dormidos y comiendo, estos son motivos por el cual los niños tienden a presentar la diabetes mellitus tipo 2 debido a que su estilo de vida no es activa dando como resultados sobrepeso, obesidad.

Plan Nutricional para el niño con Diabetes Mellitus Tipo 2

Los resultados de las encuestas realizadas a los padres de familia en relación con los grupos de alimentos se obtuvo como resultado que las cantidades correctas hacer ingeridas para el niño con diabetes son las siguientes: Carbohidratos 36\%, Proteínas 22\%, Grasas 25\%, Fibra $17 \%$.

\section{Causas}

Dentro de las causas más relevantes en la aparición de la diabetes mellitus tipo 2 es la forma de alimentarse en los niños y familiarmente teniendo un $51 \%$ de prevalencia y como consecuencia también tenemos al sobrepeso con $37 \%$. 


\section{Determinar plan nutricional en diabetes mellitus tipo 2 infantil y diseñar una guía didáctica alimentaria}

Vol. 2, núm. Esp., (2018)

Liseth Pierina Pinto López; Mercy Nathaly Alarcón Sánchez; Segundo Pacherres Seminario

\section{Conclusiones.}

De acuerdo a la muestra el mayor porcentaje de niños con diabetes se presenta en edades de 8 a 9 años, teniendo mayor influencia a los niños, esto se debe a que los padres de familia no llevan un correcto control en la alimentación del niño y más aún cuando este se encuentra dentro de la escuela, como también se debe a que los niños prefieren comer alimentos que no proporcionan nutrientes en el organismo y que son conocidos como calorías vacías.

Una de las causas principales por la cual la diabetes tiene prevalencia en el niño, es que se pudo observar que la mayoría de los niños no realiza actividad física, esto se debe al avance tecnológico, ya que los niños prefieren estar en los videojuegos, en la Tablet, en la computadora antes de realizar cualquier actividad física.

De acuerdo a los valores arrojados en la encuesta el $46 \%$ de la muestra tiene un índice glicémico de 120 - $130 \mathrm{mg} / \mathrm{dl}$ dando como resultado que existe una inapropiada alimentación en cuanto a la ingesta de carbohidratos, puesto que los niños como los padres de familia no tienen conocimiento de cuantas porciones debe consumir en el día, que tipo de preparaciones o alimentos debe evitar debido a la carga glucémica que tiene cada uno de los alimentos.

\section{Bibliografía.}

1. Pace AE, Ochoa-Vigo K. El conocimiento sobre diabetes mellitus en el proceso de autocuidado. Rev. Latino-Am. Enfermagem. 2006 Oct; 14(5): p. 728-734. 


\section{Determinar plan nutricional en diabetes mellitus tipo 2 infantil y diseñar una}

\section{guía didáctica alimentaria}

Vol. 2, núm. Esp., (2018)

Liseth Pierina Pinto López; Mercy Nathaly Alarcón Sánchez; Segundo Pacherres Seminario

2. Garcia R, Suarez R. La educación en diabetes: algunas reflexiones para la práctica. Rev Asoc Am Diabetes. 1999; 7(3): p. 170-177.

3. Reyes Sanamé FA, Pérez Álvarez ML, Ramírez Estupiñan M. Tratamiento actual de la diabetes mellitus tipo 2. Correo Científico Médico. 2016 Mar; 20(1): p. 15-20.

4. Bautista Rodríguez L, Zambrano Plata G. La calidad de vida percibida en pacientes diabéticos tipo 2. Investig Enferm. Imagen Desarr. 2015; 2(1): p. 35-39.

5. Ferreras Valentín P, Rosman L. Diabetes Mellitus. In Medicina Interna. Madrid: Elsevier; 2012. 